\title{
The Effect of Adding Kinesio Tape to Mulligan's Mobilization in Patients with A Cervicogenic Headache Lamyaa Ahmed Neyazi ${ }^{1}$, Nadia Abdelzim Fayaz ${ }^{2}$, Samah Saad Almoogy Zahran², Ahmed Hamdi Azzam
}

${ }^{2}$ Department of Physical Therapy, International Institute of Cancer, Cairo University, ${ }^{2}$ Department of Physical Therapy for Musculoskeletal Disorders and its Surgeries, Faculty of Physical Therapy, Cairo

University, ${ }^{3}$ Department of Orthopedic Surgeries, Faculty of Medicine, Cairo University, Egypt *Corresponding author: Lamya Ahmed Neyazi Farghali, Mobile: (+20) 01093435582, Email: dr.lamya3@ gmail.com

\begin{abstract}
Background: Cervicogenic headache is a major problem in many people suffering from upper cervical dysfunction with a great conflict in its physical therapy management.

Objective: The aim of the work was to determine the effect of Adding Kinesio Tape To Mulligan's Mobilization in patients with a cervicogenic headache.

Patients and methods: Fifty four patients with cervicogenic headache included in the study; from outpatient clinic, Faculty of physical therapy, Cairo University Hospital (Kasr El Ainy), were randomly assigned into two equal groups ; group A (Kinesiotaping and mulligan techniques), Group B (mulligan techniques). Their mean \pm SD age, weight, height and BMI were $37.74 \pm 5.55$ years, $80.62 \pm 6.27 \mathrm{~kg}, 170.11 \pm 5.5 \mathrm{~cm}$ and $27.48 \pm 2.73 \mathrm{~kg} / \mathrm{m}^{2}$ respectively. The visual analogue scale (VAS) is used for measuring intensity of cervicogenic headache. Frequency and duration of cervicogenic headache are collected from subjective data of patients. Correlations between the examined parameters were also measured. Kinesiotaping application with mulligan SNAGs were companied in group A and mulligan SNAGs done only in group B.

Results: There was significant improvement of VAS outcome scores and frequency and duration of cervicogenic headache in Group (A) more than Group (B).

Conclusion: It could be concluded that adding kinesio tape to mulligan's mobilization in patients with a cervicogenic headache is found to be an effective in treatment of cervicogenic headache.

Keywords: Cervicogenic headache, Kinesiotaping, SNAGs.
\end{abstract}

\section{INTRODUCTION}

A cervicogenic headache $(\mathrm{CGH})$ is a syndrome characterized by chronic hemicranial pain that is referred to the head from either bony structures or soft tissues of the neck ${ }^{(1)}$. It was first described by Sjaastad $\boldsymbol{e t}$ al. ${ }^{(2)}$, as unilateral frontotemporal headaches with clinical symptomatology similar to a migraine. $\mathrm{CGH}$ is often unilateral, but it can be bilateral. It affects mostly the occipital region, the frontal region, or the retro-orbital region. It is commonly associated with suboccipital neck pain and can be combined with ipsilateral arm discomfort ${ }^{(3)}$. The prevalence of CGH in the general population is estimated from $2,5 \%$ to $4,1 \%{ }^{(4 ; 5)}$. CGHs are thought to arise from musculoskeletal impairment(s) in the neck ${ }^{(6)}$.

Dysfunction of the atlantoaxial (C1-2) and atlantooccipital $(\mathrm{C} 0-1)$ joints have been found in $\mathrm{CGH}^{(7)}$. The relative importance of $\mathrm{C} 1-\mathrm{C} 2$ as a primary cause of cervicogenic headache is also supported by Aprill et al. ${ }^{(8)}$. Zito et al. (9) have confirmed the importance of examination of the $\mathrm{C} 1$ $\mathrm{C} 2$ segment in $\mathrm{CGH}$ diagnosis. Limitations in cervical muscle strength, endurance, and control have been associated with $\mathrm{CGH}^{(\mathbf{1 0})}$.

On manual examination of a patient with $\mathrm{CGH}$, Moore ${ }^{(11)}$ found a weakness of deep neck flexors and tightness of upper trapezius, levator scapulae, and sternocleidomastoid muscles. Kinesio taping (KT) method is a somewhat new type of taping technique. It was originally created by a Japanese chiropractor, Kenzo Kase in 1980 and has gained popularity in the clinical setting. KT is an effective method for decreasing tightness and pain intensity in soft tissues ${ }^{(\mathbf{1 2})}$.

Kilinç et $\boldsymbol{a l} .^{(\mathbf{1 3 )}}$ found that KT application had the same effect of the mobilization techniques on decreasing the neck pain, headache intensity and enhancing the activation of deep cervical neck flexor muscles in mechanical neck problems. Falla et al. ${ }^{(14)}$ found that KT improved the muscular endurance of deep neck flexors. KT increased the muscle activation of back extensor muscles ${ }^{(\mathbf{1 5})}$.

One of the techniques that has been used in managing CGH is Sustained Natural Apophyseal Glides (SNAGS) which involve a combination of a sustained facet glide with active motion, which is then followed by overpressure ${ }^{(16)}$. The efficacy of the Mulligan concept was demonstrated in reducing of CGH symptoms and improving cervical range of movement. The explanation of reducing headache symptoms is neuromodulation effect of joint mobilization ${ }^{(17)}$.

The purpose of this randomized controlled study was to investigate the effectiveness of adding 
KT to Mulligan's mobilization in patients with a cervicogenic headache.

\section{MATERIALS AND METHODS}

This study included a total of 54 patients with cervicogenic headache, attending at outpatient clinic, Faculty of physical therapy, Cairo University Hospital (Kasr El Ainy).

\section{Ethical approval:}

Subjects in this study were informed about the study procedure and signed a written informed consent. Approval of the ethical committee of Cairo University was obtained.

The included subjects were randomly divided into two equal groups, 27 each; Group A were received KT and Mulligan's mobilization, Group B were received mulligan's mobilization. The treatment was set for 3 sessions per week for 6 weeks ${ }^{(\mathbf{1 8}, \mathbf{1 9})}$.

To ensure the randomization process we had used random number generator with blocks software program randomization.

\section{Inclusion Criteria:}

Patients were selected to be enrolled into this study after they had fulfilled the inclusion criteria of the study; age range from 20-50years, unilateral or a side-dominant headache without side shift, had at least one episode in previous 3 months. Positive flexion-rotation test and restriction greater than $10^{\circ}(20)$.

\section{Exclusion Criteria:}

Subjects were excluded if they had a headache not of cervical origin .Physiotherapy or chiropractic treatment in the past 3 months, a headache with autonomic involvement, dizziness, or visual disturbance. Congenital conditions of the cervical spine. Contraindication to manipulative therapy. Involvement in litigation or compensation. Inability to tolerate the flexion-rotation test ${ }^{(20)}$.

Instrumentation: Instrumentation used for evaluation: patient was assessed just before and just after the treatment program. The assessment procedure was included the following:

Visual Analogue Scale (VAS): is a graphical descriptive scale, where the test determines the degree of pain intensity on a line with a length of 10 $\mathrm{cm}$ (21). The VAS is a tool for measuring musculoskeletal pain with excellent reliability and validity (22).

Subjective questionnaire: patients were asked about duration and frequency of cervicogenic headache.

\section{Evaluation procedure:}

Visual Analogue Scale (VAS): this scale allows continuous data analysis and use a $10 \mathrm{~cm}$ line with 0 (no pain) and 10 (killing pain), each patient was assessed before and after treatment program (six weeks) and comparing the results.

Subjective questionnaire: patients were asked about duration and frequency of cervicogenic headache.

\section{Instrumentation used for treatments:}

Kinesio Tape (KT): An adhesive, super-rigid Tape, primarily used for its improvements in neuromuscular re-education ${ }^{(23)}$. This technique is based on the body's on natural healing process; that has been used to assist in correcting muscle function, improving circulation of blood and lymph, relieving pain. Reducing edema and repositioning the subluxation joints ${ }^{(24)}$.

\section{Mulligan's SNAG technique:}

Mulligan proposed that injuries or sprains might result in a minor positional fault to a joint thus causing restrictions in physiological movement. Unique to this concept is the mobilization of the spine whilst the spine is in a weight bearing position and directing the mobilization parallel to the spinal facet planes ${ }^{\mathbf{2 5})}$.

Mulligan proposed that when an increase in the pain-free range of movement occurs with a SNAG it is primarily the correction of a positional fault at the zygapophyseal joint, although a SNAG also influences the entire spinal functional unit (SFU).

\section{Treatment procedures:}

Group A: were received KT and Mulligan's mobilization The patient was in sitting position and therapist was standing behind the patient. Examiner applied KT on upper fibers of trapezius and deep cervical extensors muscles ${ }^{(\mathbf{1 8})}$. The tape was kept in place and changed weekly. It was applied on deep cervical extensors by measuring the distance between the occipital union and T4/T5 cervical vertebrae; two pieces of the tape was cut in a Yshape equal to this distance.

The base was fixed at T4/T5, and the two strips of the tape were run along the spine, one on the right side and the other on the left side. Patients were then asked to flex the cervical vertebrae (maximum flexion) while making a heterolateral rotation of the head, and the tape was stretched, and the anchor was fixed below the occipital union. We then put pressure by the knuckles on the tape to stimulate its adhesive effect ${ }^{(\mathbf{1 8})}$. For the upper fibers of the trapezius, we measured the distance between 
the acromion processes and the occipital union. We cut two pieces of tape equal to this distance. We started by fixing the base of the tape on the origin of upper fibers of the trapezius. Then, the patients were asked to laterally flex the neck (maximum side bending), and we fixed the anchor at the insertion in the acromion process. We then put pressure by the knuckles on the tape to stimulate its adhesive effect (18).

\section{Mulligan's SNAG technique:}

The patient was in sitting position and therapist was standing behind the patient. Examiner placed the thumb on the spinous process of the $\mathrm{C} 1-\mathrm{C} 2$ Therapist then moved the spinous process upward towards eyeball direction and maintain this glide and ask the patient to turn (rotation) his head slowly in restricted painful direction, sustain the mobilization until head returns to the midline ${ }^{(26)}$. Four repetitions of each glide were given and were maintained for 10 seconds at end range or the onset of pain ${ }^{(27)}$.

Group B: were received mulligan's mobilization the patient was in sitting position and therapist was standing behind the patient. Examiner placed the thumb on the spinous process of the C1-C2. Therapist then moved the spinous process upward towards eyeball direction and maintain this glide and ask the patient to turn (rotation) his head slowly in restricted painful direction, sustain the mobilization until head returns to the midline ${ }^{(26)}$. Four repetitions of each glide were given and were maintained for 10 seconds at end range or the onset of pain ${ }^{(27)}$.

\section{Statistically analysis}

Subject characteristics were compared between groups using t-test. Chi- squared test was used for comparison of sex distribution between groups. $t$ test was conducted to compare mean values of headache pain intensity, frequency and duration between both groups; and paired t test was conducted to compare between pre and post treatment mean values of the measured variables in each group. The level of significance for all statistical tests was set at $\mathrm{p}<0.05$. All statistical analysis was conducted through the statistical package for social studies (SPSS) version 19 for windows (IBM SPSS, Chicago, IL, USA).

\section{RESULTS \\ Subject characteristics:}

Table 1 showed the subject characteristics of both groups. There was no significant difference between both groups in the mean age, weight, height and BMI $(\mathrm{p}>0.05)$. Also, there was no significant difference in sex distribution between groups ( $\mathrm{p}>$ $0.5)$.

Table (1): Comparison of subject characteristics between group A and B.

\begin{tabular}{|llllll|}
\hline & Group A & Group B & & & \\
\cline { 2 - 3 } Age $($ years $)$ & Mean \pm SD & Mean \pm SD & MD & t- value & P-value \\
\cline { 2 - 3 } Weight $(\mathrm{kg})$ & $38.46 \pm 3.71$ & $38 \pm 4.19$ & 0.46 & 0.32 & $0.74^{*}$ \\
Height $(\mathrm{cm})$ & $80.13 \pm 7.67$ & $78.93 \pm 6.68$ & 1.2 & 0.45 & $0.65^{*}$ \\
BMI $\left(\mathrm{kg} / \mathrm{m}^{2}\right)$ & $170.26 \pm 6$ & $167.86 \pm 6.34$ & 2.4 & 1.06 & $0.29^{*}$ \\
Males/females & $27.71 \pm 3.05$ & $27.98 \pm 1.41$ & -0.27 & -0.31 & $0.75^{*}$ \\
\hline
\end{tabular}

$\mathrm{x}$, Mean; SD, Standard deviation; MD, Mean difference; $\chi 2$, Chi squared value; $\mathrm{p}$ value, Probability value; *, Non-significant.

\section{- Effect of treatment on headache pain intensity, frequency, and duration: \\ - Within group comparison:}

There was a significant decrease in headache pain intensity, headache frequency and headache duration in group A compared with that pre-treatment $(\mathrm{p}=0.0001)$; with the precent of decrease in pain, frequency and duration were $62.5,56.79$ and $65.26 \%$ respectively (Table 2 ).

Regarding group B, there was a significant decrease in headache pain intensity, headache frequency and headache duration compared with that pre-treatment $(\mathrm{p}=0.0001)$; with the precent of decrease in pain, frequency and duration were $37.6,32.84$ and $30.76 \%$ respectively (Table 2).

\section{Comparison between groups:}

There was no significant difference between both groups in all variables pre-treatment $(\mathrm{p}>0.05)$. Comparison between groups post treatment revealed a significant decrease in headache pain intensity, headache frequency and headache duration of group A compared with that of group B $(\mathrm{p}<0.05)$ (Table 3$)$. 
Table (2): Mean headache pain intensity, frequency and duration pre and post treatment of group A and B:

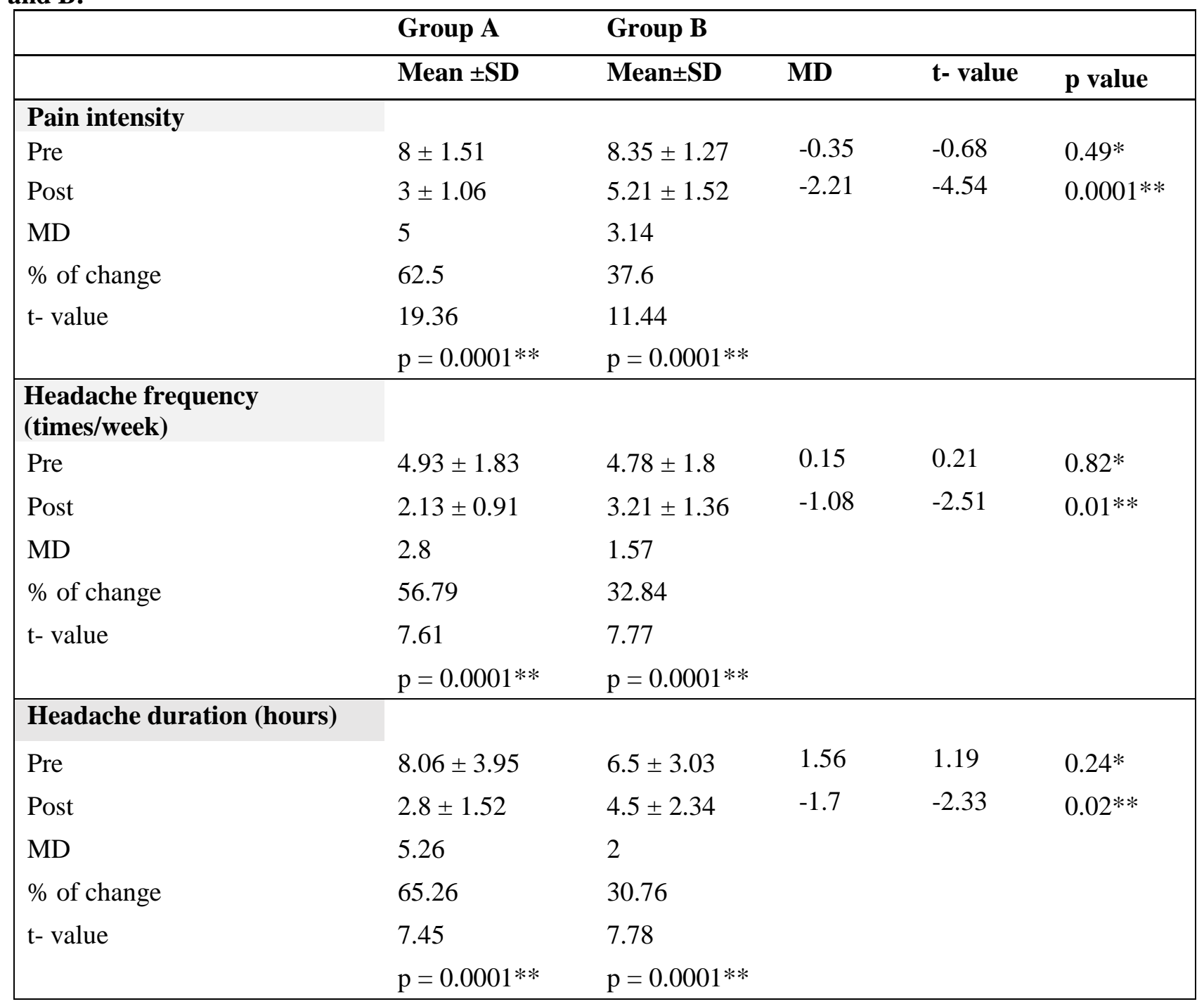

$\mathrm{x}$, Mean; SD, Standard deviation; MD, Mean difference; $\mathrm{p}$ value, Probability value; *, Non-Significant ; **, Significant

\section{DISCUSSION}

This study was conducted to identify the effect of adding kinesio Tape to Mulligan's Mobilization in patients with cervicogenic headache.

The results of the present study showed that combing of kinesio tape and mulligan tehcnique more than one protocol of treatment was improved and effective in cervicogenic hgeadache than single protocol mulligan teqhnique.The main findings of the study confirmed that kinesiotaping with selected physical therapy program for one month has a psotive effect of cerviogenic headache as resuls of the present study revelaed that there was significant difference in the VAS, frequancy and duration of cervicogenic headache between study and control group ${ }^{(28)}$. The results of Ewa and Carol Showed that Tapin can be used as an adjunct during the rehabilitation program to enhance fucntinal recovery by reduing pain, imrpoving alignment, and stimulating or inhibiting muscle fucntion and improving properioceptive fucntion of the joint structures ${ }^{(29,30)}$.

Results we have concluded in the current study was supported by results of Shin and Lee ${ }^{(31)}$ and Hall et $\boldsymbol{a l} .{ }^{(20)}$ who studied specifically SNAGs mobilization technique on $\mathrm{C} 1-\mathrm{C} 2$ and its effect on cervicognenic headache where shin and Lee found that the SNAGs intervention group had greater reductions in disability, intensity, and duration than the control group. Additionally, a study done by Khan et al. (32) who compared SNAGs with posterior anterior vertaebral mobilization (PAVM) in treating $\mathrm{CGH}$, their research revealed that although both groups had improvements in neck disability index (NDI) and visual analog (VAS) scores, the cervical SNAGs treatment group was more effective for both NDI and VAS. These findings indicate some mobilizations or manipulations may have greater efficacy than others in reducing $\mathrm{CGH}$ symptoms. The possible mechanism behind the effectiveness of SNAGs 
techniques on varibles like pain intensity and headache symptoms is that mobilizations stimulate mechnaoreceptors exist in upper cervical facet joints to inhibit pain in spinal cord by activationg gate control theory as reported by ${ }^{(33)}$. End rang or rotation moveent improvement following SNAGs mobilzation might be the cause of engaging descending inhibitory pain mechanism which could be mediated and activated by areas of preaquiductal grey of mid-brain as Sterling et al. ${ }^{(34)}$ had said.

Out results in this study agreed with results of Saleh et al. ${ }^{(18)}$ that showed potentiation of physictherapy by low level laser or kinesio taping for treatment of cervicogenic headache

On the other hand Added et al. ${ }^{(35)}$ which showed that Kinesio Taping does not provide additional benefits in patients with chronic low back pain who receive exercise and manual therapy.

The current study showed that adding kinesio tape to mulligan's mobilization in patients with a cervicogenic headache get better results than mulligan's mobilization only.

\section{CONCLUSION}

It could be concluded that adding kinesio tape to mulligan's mobilization in patients with a cervicogenic headache is found to be an effective in treatment of cervicogenic headache.

\section{RECOMMENDATIONS}

A Similar study should be conducted on a large number of patients to provide a wide representation of the data. Further studies should be done for using other physical therapy interventions that improve cervicogenic headache. Similar studies should be conducted dependent on gender factor as the incidence of cervicogenic headache is bigger in female population. EMG physiological studies are recommended to be applied on sub-occipital muscles to see the effect of different types of modalities on its physiological state in cervicogenic headache population.

\section{REFERENCES}

1. Biondi D (2005): Cervicogenic headache: a review of diagnostic and treatment strategies. J Am Osteopath Assoc., 105: 16-22.

2. Sjaastad O, Saunte C, Hovdahl H et al. (1983): 'Cervicogenic' headache: a hypothesis. Cephalalgia, 3: 249-56.

3. Jensen R, Stovner L (2008): Epidemiology and comorbidity of headache. Lancet Neurol., 7:354-61.

4. Haldeman S, Dagenais S (2001): Cervicogenic headaches: a critical review. Spine J., 1(1): 31-46.

5. Martelletti P, van Suijlekom H (2004): Cervicogenic headache: practical approaches to therapy. CNS Drugs, 18: 12: 793-805.
6. Jull G (1997): Management of cervical headache. Man Ther., 2:182-90.

7. Dreyfuss P, Michaelsen M, Fletcher D (1994): Atlanto-occipital and lateral atlanto-axial joint pain patterns. Spine, 19:1125-31.

8. Aprill C, Axinn M, Bogduk N (2002): Occipital headaches stemming from the lateral atlantoaxial (C1-C2) joint. Cephalalgia., 22:15-22.

9. Zito G, Jull G, Story I (2006): Clinical tests of musculoskeletal dysfunction in the diagnosis of cervicogenic headache. Man Ther., 11: 118-129.

10. Barton P, Hayes K (1996): Neck flexor muscle strength, efficiency, and relaxation times in normal subjects and subjects with unilateral neck pain and headache. Arch Phys Med Rehabil., 77(7):680-687.

11. Moore M (2004): Upper cross syndrome and its relationship to cervicogenicheadache. Journal of Manipulative Physiol Ther., 27:414-20.

12. Karatas N, Bicici S, Baltaci $G$ et al. (2012): The effect of Kinesiotape application on functional performance in surgeons who have musculoskeletal pain after performing surgery. Turk Neurosurg., 22(1):83-9.

13. Kılınç H, Harput G, Baltacı G (2015): Additional Effects of Kinesiotaping to Mobilization Techniques in Chronic Mechanical Neck Pain, Turk J Physiother Rehabil., 26(3):107-113

14. Falla D, Jull G, Russell T et al. (2007): Effect of neck exercise on sitting posture in patients with chronic neck pain. Physical Therapy, 87(4):408-17.

15. Alvarez-Alvarez $S$, Jose $F$, Rodriguez-Fernandez $A$ et al. (2014): Effects of Kinesio Tape in low back muscle fatigue: Randomized, controlled, doubledblinded clinical trial on healthy subjects. J Back Musculoskelet Rehabil., 27(2):203- 12.

16. Mulligan B (2004): Manual therapy: NAGS, SNAGS, MWMS etc. (5th Ed). Plane View Services Ltd. https://www.amazon.com/Manual-Therapy-NAGSSNAGS-MWMS/dp/047601154X

17. Kocjan J (2015): Effect of a C1-C2 Mulligan sustained natural apophyseal glide (SNAG) in the treatment of cervicogenic headache. Journal of Education, Health, and Sport, 5(6):79-86.

18. Saleh H, Edward M, Abdel-Fattah A et al. (2016): Potentiation of physiotherapy by low-level laser or kinesiotaping for treatment of cervicogenic headache. The Egyptian Journal of Otolaryngology, 32:248-254.

19. Alanzy L, Alanzy G, Raafat M (2017): Kinesio Taping In Management Of Cervicogenic Headache. World Journal of Pharmacy and Pharmaceutical Sciences, 6:328- 337.

20. Hall T, Briffa K, Hopper D (2007): Clinical Evaluation of Cervicogenic Headache: A Clinical Perspective. J Man Manip Ther., 16(2): 73-80.

21. Sip P, Sip N, Manikowski W (2013): The usefulness of kinesiotaping method to reduce the activity of myofascial trigger points in trapezius muscle. Issue of Rehabilitation, Orthopaedics, Neurophysiology and Sport Promotion, 4: 11-17.

22. Boonstra A, Preuper S, Reneman M et al. (2008): Reliability and validity of the visual analogue scale for disability in patients with chronic musculoskeletal pain. Int J Rehabil Res., 31: 165-169. 
23. Osterhues D (2004): The use of $\mathrm{KT}$ in the management of traumatic patella dislocation: a case study. An International Journal of Physical Therapy, 20:267-270.

24. Kaya E, Zinnuroglu M, Tugcu I (2011): Kinesio taping compared to physical therapy modalities for the treatment of shoulder impingement syndrome. Clin Rheumatol., 30(2):201-7.

25. Mulligan B (1999): Manual therapy "Nags", "Snags", "MWMs"'etc., 4th Edn. Plane View Services, Wellington, New Zealand, Pp. 1-144.

26. Schoensee S, Jenson G, Nicholson G et al. (1995): The effect of mobilization on cervical headaches. JOSPT., 21:18496.

27. D'Sylva J, Miller J, Gross A et al. (2010): Manual therapy with or without physical medicine modalities for neck pain: a systematic review. Man Ther., 15(5): 415-433.

28. Alix M, Bates D (1999): A Proposed Etiology of Cervicogenic Headache: The Neurophysiologic Basis and Anatomic Relationship Between the Dura Mater and the Rectus Posterior Capitis Minor Muscle J Manipulative Physiol Ther., 22 (8): 534-539.

29. Jaraczewska E, Long C (2006): Kinesio taping in stroke: improving functional use of the upper extremity in hemiplegia. Top Stroke Rehabil.,13(3):31-42.
30. Aboshady O, Radwan A, Eltaweel A et al. (2015): Perception and use of massive open online courses among medical students in a developing country: multicentre cross-sectional study. BMJ Open, 5(1):6804-8.

31. Shin E, Lee B (2014): The effect of sustained natural apophyseal glides on headache, duration and cervical function in women with cervicogenic headache. Journal of Exercise Rehabilitation, 10(2): 131-135.

32. Khan M, Ali S, Soomro R (2014): Efficacy of C1-C2 Sustained Natural Apophyseal Glide (SNAG) Versus Posterior Anterior VertebralMobilization (PAVMs) in the Management of Cervicogenic Headache. Journal of Basic \& Applied Sciences, 10: 226-230.

33. Wright A (1995): Hypoalgesia post -manipulative therapy : a review of apotential neurophysiological mechanism. Man Ther., 1: 11-16.

34. Sterling M, Jull G, Wright A (2001): Cervical mobilisation: concurrent effects on pain, sympathetic nervous system activity and motor activity. Man Ther., 6(2):72-81.6(2): 72-81.

35. Added M, Costa L, De Freitas D et al. (2016). Kinesio Taping does not provide additional benefits in patients with chronic low back pain who receive exercise and manual therapy: a randomized controlled trial. Journal of Orthopaedic \& Sports Physical Therapy, 46(7): 506-513. 